\title{
Numerical Study of a Thermal Convection Induced by a Purely Internal Heating in a Rotating Medium Saturated by a Radiating Nanofluid
}

\author{
Abderrahim Wakif \\ Hassan II University, \\ Laboratory of Mechanics, \\ Casablanca, Morocco
}

\author{
Zoubair Boulahia \\ Hassan II University, \\ Laboratory of Mechanics, \\ Casablanca, Morocco
}

\author{
Rachid Sehaqui \\ Hassan II University, \\ Laboratory of Mechanics, \\ Casablanca, Morocco
}

\begin{abstract}
In this paper, we use the Buongiorno's mathematical model for studying numerically the convective instability which is induced by a purely internal heating in a rotating medium confined between two isothermal surfaces and filled of a Newtonian nanofluid layer (water + alumina) in the case where the radiation heat transfer mode is taken into consideration, such that the nanoparticle flux is zero on the boundaries. The linear study which was achieved in this investigation shows that the thermal stability of nanofluids depends of the Coriolis forces generated by the rotation of the system, the thermal radiation parameter, the Brownian motion, the thermophoresis of nanoparticles and other thermophysical properties of nanoparticles. The studied problem will be solved by converting our boundary value problem to an initial value problem, after this step we will approach numerically the searched solutions using the power series method (PSM).
\end{abstract}

\section{Keywords}

Convection, Thermal radiation, Purely internal heating, Rotation, Brownian motion, Thermophoresis.

\section{INTRODUCTION}

The nanofluid is defined as a homogeneous fluid in which nano-sized particles (nanoparticles) are suspended in a base fluid like water, ethylene glycol and oil. The nanoparticles used in nanofluids are generally prepared of metals, oxides, carbides, or carbon nanotubes. The purpose of using nanofluids is to obtain a higher value of heat transfer coefficient compared with that of the base fluid, such that the nanofluids have not only a better heat conductivity but also greater convective heat transfer capability than that of base fluids, this remarkable properties make them potentially useful in many practical applications. Currently the nanofluids are introduced in the solar collectors instead of using conventional fluids for increasing the efficiency of the heat transfer.

The onset of Rayleigh-Bénard convection in a confined medium filled of a nanofluid layer heated from below is now regarded as a classical problem when the volumetric fraction of nanoparticles is constant at the horizontal walls limiting the layer, such that the buoyancy-driven convection occurs when the Rayleigh number exceeds a certain critical value which we can determine it numerically with a good accuracy. In the recent years, the precedent problem has been studied without considering the radiation heat transfer mode in different situations by several authors [1-7] using the Galerkin weighted residuals method based on well-defined test functions, they found that the critical Rayleigh number can be decreased or increased by a significant quantity depending on the relative distribution of nanoparticles between the top and bottom walls. Combined the heat transfer processes such as convection - radiation play a significant role in several chemical processes involving combustion, drying, fluidization , MHD flows, and so forth.

The problem of radiative-convective flows for the fluids has been studied by several authors [8-20] in different situations and geometrical configurations. Goody [8] found an approximate solution of the problem of the onset of convection between plane-parallel plates heated from below when the fluid between them absorbs and emits the thermal radiations, he developed the equation of radiative transfer in two approximate forms, one appropriate to an opaque medium, the other to a transparent medium .Vedat S. Arpaci [9] studied the non-equilibrium interaction between thermal radiation and laminar free convection in terms of a heated vertical plate in a stagnant radiating gas . C. Christophorides and Stephen H. Davis [10] studied the effects of radiative transfer in the thermal of a thin fluid layer. J. C. Bratis and J.R. Novotny [11] reported on the effects of thermal radiation in the convection boundary layer-regime of an enclosure. C.Chang et al. [12] used a radiative flux diffusion approximation to model the interaction of convective and radiative heat transfer in two-dimensional complex enclosure. Wen-Mei Yang [13] studied numerically the thermal instability of a fluid layer above a solid boundary induced by incident radiative heat to the upper free surface. F. Bdéoui and A. Soufiani [14] studied theoretically the radiation effect on the onset of Rayleigh-Bénard instability in the case of real molecular emitting and absorbing gases. A. Raptis [15] discussed the effects of the radiation on the velocity field in the case of the free convection flow through a very porous medium bounded by a vertical infinite porous plate. M.A. Hossain et al. [16] used the implicit finite difference method to study the flow and heat transfer characteristics of laminar combined forced and free convection, with the effects of radiation, of a viscous incompressible and optically dense fluid from a horizontal cylinder. A. J. Chamkha [17] studied numerically the combined influence of radiative flux, gravity field and heat absorption on convection heat transfer in a twophase flow. M.Anwar Hossain et al. [18] investigated numerically the effect of thermal radiation on the natural convection flow along a uniformly heated vertical porous plate with variable viscosity and uniform suction velocity in the case where the fluid is considered as an optically dense viscous incompressible fluid of temperature-dependent viscosity. Vincent E. Larson [19] studied analytically the linear and nonlinear stability properties of an idealized radiative-convective model due to Goody, he used the energy method to rule out subcritical instabilities in the case where the thermal diffusivity is zero, if not he used this method to find a critical threshold below which all infinitesimal and finite-amplitude perturbations are stable in the case where the 
thermal diffusivity is nonzero. P.Ganesan and P. Loganathan [20] analyzed the interaction of free convection with thermal radiation of a viscous incompressible unsteady flow past a moving vertical cylinder with heat and mass transfer.

In general, the radiative process either occurs at the boundaries or as a term in the energy equation. In the latter case, the radiative term is usually approximated as a flux in such a way that the term corresponding to radiation in the heat transfer equation appears as a gradient term similar to Fourier's conduction term.

Today, the problem of Rayleigh-Benard convection for the nanofluids is studied by some authors [21-28] in different situations (with or without: rotation, magnetic field, internal heat source ...) using a new type of boundary conditions for the nanoparticles which combines the contribution of the Brownian motion and the thermophoresis of nanoparticles instead to impose a nanoparticle volume fraction at the boundaries of the layer. The new model of boundary conditions assumes that the nanoparticle flux must be zero on the impermeable boundaries. D.A. Nield and A.V. Kuznetsov [21] are considered as the first ones who were used this type of boundary conditions for the nanoparticles. Until now, the precedent boundary conditions are used to study the problem of natural convection in a porous (Darcy or Brinkman model) or non-porous medium saturated by a nanofluid in the case where the radiation heat transfer mode is neglected.

Our work consists of studying numerically the RayleighBénard problem in a rotating medium confined between two isothermal rigid-rigid boundaries and filled of a nanofluid layer (water + alumina) in the case where the convective instability is induced by a purely internal heating and the energy transfer by radiation is present, such that the nanoparticle flux is assumed to be zero on the boundaries. In this investigation we assume that the Rosseland approximation (R. Viskanta and R. J. Grosh [29]) is valid and the nanofluid is Newtonian, gray-emitting and absorbing radiation but non-scattering. The parameters which appear in the governing equations are considered constant in the vicinity of the temperature of the boundaries $T_{0}^{*}$ which we took it as a reference temperature. Finally we will impose that the flow is laminar and the radiation heat transfer mode between the horizontal walls is non negligible. Our problem will be solved with a more accurate numerical method based on analytic approximations (power series method). In this investigation we assume that the effect of the rotation in the momentum equation is restricted to the Coriolis force and also the centrifugal acceleration is negligible compared to the buoyancy force. The used method gives results with an absolute error of the order of $10^{-3}$ to the critical values characterizing the onset of the convection. To show the accuracy of our method in this study, we will check some results treated by $\mathrm{Yu}$ and Shih [30] concerning the onset of convective instability which is induced by a purely internal heating in a non-rotating layer of a regular fluid confined between two isothermal rigid-rigid boundaries without taking into account the radiation heat transfer mode.

\section{MATHEMATICAL FORMULATION}

We consider a dilute layer saturated by an incompressible Newtonian nanofluid (water + alumina), vertically confined between two parallel rigid impermeable boundaries (Fig 1) where the temperature is constant and the nanoparticle flux is zero on the boundaries, such that:

$$
\left\{\begin{array}{l}
\mathrm{w}^{*} \mathrm{~J}_{\mathrm{z}^{*}=0 ; 1}=\left.\frac{\partial \mathrm{w}^{*}}{\partial \mathrm{z}^{*}}\right|_{\mathrm{z}^{*}=0 ; \mathrm{L}}=\left.\left(\frac{\partial \mathrm{v}^{*}}{\partial \mathrm{x}^{*}}-\frac{\partial \mathrm{u}^{*}}{\partial \mathrm{y}^{*}}\right)\right|_{\mathrm{z}^{*}=0 ; \mathrm{L}}=0 \\
\mathrm{~T}^{*} \mathrm{~J}_{\mathrm{z}^{*}=0 ; \mathrm{L}}=\mathrm{T}_{0}^{*} \\
\left.\left(\mathrm{D}_{\mathrm{B}} \frac{\partial \mathrm{\chi}^{*}}{\partial \mathrm{z}^{*}}+\frac{\mathrm{D}_{\mathrm{T}}}{\mathrm{T}_{0}^{*}} \frac{\partial \mathrm{T}^{*}}{\partial \mathrm{z}^{*}}\right)\right|_{\mathrm{z}^{*}=0 ; \mathrm{L}}=0
\end{array}\right.
$$

In this investigation we consider that the nanoparticles are spherical, their concentration is low and the convection is due to a uniform internal heating of strength $Q_{s}^{*}$ where the nanofluid is assumed to be gray-emitting and absorbing radiation but non-scattering. The radiative heat flux in the $x^{*}$ and $y^{*}$ directions is considered negligible in comparison to that in the $\mathrm{z}^{*}$ direction and the nanofluid will be subjected to a uniform rotation characterized by an angular velocity $\vec{\Omega}^{*}$ and also acted upon by the gravity force $\vec{g}^{*}$.The thermo-physical properties of nanofluid (viscosity, thermal conductivity, specific heat, mean absorption coefficient ) are assumed constant in the vicinity of the reference temperature $T_{0}^{*}$ except for the density variation in the momentum equation which is based on the Boussinesq approximations .The asterisks are used to distinguish the dimensional variables from the nondimensional variables (without asterisks).

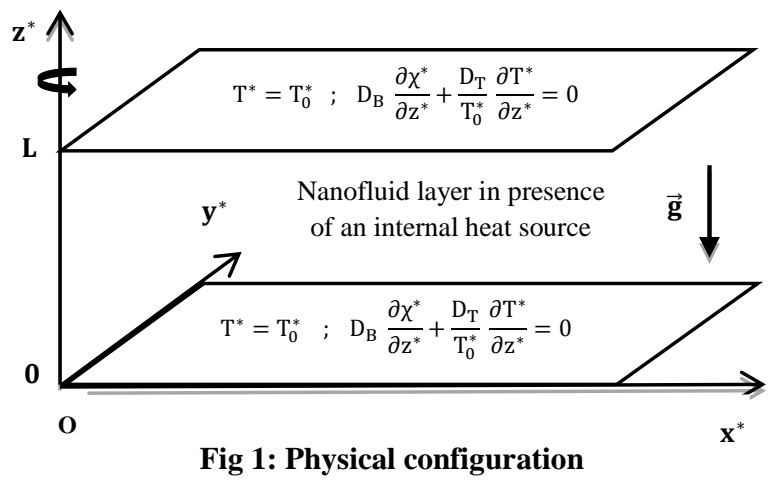

The governing equations for nanofluids given by Buongiorno [1] and Tzou [2,3] are extended under this model are as follows:

$$
\begin{gathered}
\vec{\nabla}^{*} \cdot \overrightarrow{\mathrm{V}}^{*}=0 \\
\rho_{0}\left[\frac{\partial \overrightarrow{\mathrm{V}}^{*}}{\partial \mathrm{t}^{*}}+\left(\overrightarrow{\mathrm{V}}^{*} \cdot \vec{\nabla}^{*}\right) \overrightarrow{\mathrm{V}}^{*}\right]=-\vec{\nabla}^{*} \mathrm{P}^{*}-2 \rho_{0} \vec{\Omega}^{*} \times \overrightarrow{\mathrm{V}}^{*}+\mu \vec{\nabla}^{*} \overrightarrow{\mathrm{V}}^{*} \\
+\left\{\rho_{0}\left[1-\beta\left(\mathrm{T}^{*}-\mathrm{T}_{0}^{*}\right)\right]\left(1-\chi^{*}\right)+\rho_{\mathrm{p}} \chi^{*}\right\} \overrightarrow{\mathrm{g}}^{*} \\
(\rho \mathrm{c})\left[\frac{\partial \mathrm{T}^{*}}{\partial \mathrm{t}^{*}}+\left(\overrightarrow{\mathrm{V}}^{*} \cdot \vec{\nabla}^{*}\right) \mathrm{T}^{*}\right]=\kappa \vec{\nabla}^{*} \mathrm{~T}^{*}-\frac{\partial \mathrm{q}_{\mathrm{r}}^{*}}{\partial \mathrm{z}^{*}}+\mathrm{Q}_{\mathrm{s}}^{*} \\
+(\rho \mathrm{c})_{\mathrm{p}}\left[\mathrm{D}_{\mathrm{B}} \vec{\nabla}^{*} \chi^{*} \cdot \vec{\nabla}^{*} \mathrm{~T}^{*}+\left(\frac{\mathrm{D}_{\mathrm{T}}}{\mathrm{T}_{0}^{*}}\right) \vec{\nabla}^{*} \mathrm{~T}^{*} \cdot \vec{\nabla}^{*} \mathrm{~T}^{*}\right] \\
\frac{\partial \chi^{*}}{\partial \mathrm{t}^{*}}+\left(\overrightarrow{\mathrm{V}}^{*} \cdot \vec{\nabla}^{*}\right) \chi^{*}=\mathrm{D}_{\mathrm{B}} \vec{\nabla}^{*} \chi^{2}+\left(\frac{\mathrm{D}_{\mathrm{T}}}{\mathrm{T}_{0}^{*}}\right) \vec{\nabla}^{*} \mathrm{~T}^{*}
\end{gathered}
$$

Where $\vec{V}^{*}\left(u^{*}, v^{*}, w^{*}\right)$ is the nanofluid velocity, $t^{*}$ is the time, $\rho_{0}$ is the nanofluid density at reference temperature $T_{0}^{*}, P^{*}$ is the pressure, $\rho_{p}$ is the density of nanoparticles, $\beta$ is the thermal expansion coefficient, $\chi^{*}$ is the volumetric fraction of nanoparticles, $\mathrm{c}$ is the specific heat $, \mu, \rho$ and $\kappa$ are the viscosity, the density and the thermal conductivity of nanofluid respectively, $D_{B}=k_{B} T_{0}^{*} / 3 \pi \mu d_{p}$ is the Brownian diffusion coefficient , $D_{T}=\tilde{\beta} \mu \chi^{*} / \rho_{0}$ is the thermophoretic diffusion coefficient, $k_{B}$ is the Boltzmann's constant, $d_{p}$ is the diameter of nanoparticles , $\tilde{\beta}$ is a thermal coefficient $\left(\tilde{\beta}=0.26 \kappa /\left(2 \kappa+\kappa_{\mathrm{p}}\right)\right), \kappa_{\mathrm{p}}$ is the thermal conductivity of nanoparticles, $\vec{\nabla}^{*}$ is the vector differential operator and $q_{r}^{*}$ is the radiation heat flux.

In order to reduce the complexity of the problem and to provide a mean of comparison with the previous studies, the 
optically thick radiation limit is considered in the present analysis. Thus, the radiative heat flux term is simplified by using the Rosseland approximation [29] as:

$$
\mathrm{q}_{\mathrm{r}}^{*}=-\frac{4 \sigma_{\mathrm{e}}}{3 \beta_{\mathrm{R}}} \frac{\partial \mathrm{T}^{* 4}}{\partial \mathrm{z}^{*}}
$$

Where $\sigma_{e}$ and $\beta_{R}$ are respectively the Stefan-Boltzmann constant and the mean absorption coefficient.

Assuming that the temperature gradient is sufficiently small in the medium, the Taylor's series for $T^{* 4}$ about the reference $T_{0}^{*}$ allows us to write:

$$
\mathrm{T}^{* 4}=\mathrm{T}_{0}^{* 4}+\sum_{\mathrm{i}=1}^{+\infty} \frac{\left(\mathrm{T}^{*}-\mathrm{T}_{0}^{*}\right)^{\mathrm{i}}}{\mathrm{i} !} \times\left.\frac{\partial^{\mathrm{i}}}{\partial \mathrm{T}^{* \mathrm{i}}}\left(\mathrm{T}^{* 4}\right)\right|_{\mathrm{T}^{*}=\mathrm{T}_{0}^{*}}
$$

If we neglect the higher order terms, the function $T^{* 4}$ may be expressed as a linear function of the temperature $T^{*}$ to obtain $T^{* 4}=4 T_{0}^{* 3} T^{*}-3 T_{0}^{* 4}$, then the equation (3) becomes:

$$
\begin{aligned}
&(\rho c)\left[\frac{\partial \mathrm{T}^{*}}{\partial \mathrm{t}^{*}}+\left(\overrightarrow{\mathrm{V}}^{*}\right.\right.\left.\left.\cdot \vec{\nabla}^{*}\right) \mathrm{~T}^{*}\right]=\kappa \vec{\nabla}^{*}{ }^{2} \mathrm{~T}^{*}+\frac{16 \sigma_{\mathrm{e}} \mathrm{T}_{0}^{* 3}}{3 \beta_{\mathrm{R}}} \frac{\partial^{2} \mathrm{~T}^{*}}{\partial \mathrm{z}^{* 2}}+\mathrm{Q}_{\mathrm{s}}^{*} \\
&+(\rho \mathrm{c})_{\mathrm{p}}\left[\mathrm{D}_{\mathrm{B}} \vec{\nabla}^{*} \chi^{*} \cdot \vec{\nabla}^{*} \mathrm{~T}^{*}+\left(\frac{\mathrm{D}_{\mathrm{T}}}{\mathrm{T}_{0}^{*}}\right) \vec{\nabla}^{*} \mathrm{~T}^{*} \cdot \vec{\nabla}^{*} \mathrm{~T}^{*}\right]
\end{aligned}
$$

Introducing the following dimensionless variables:

$$
\begin{aligned}
& \left(\mathrm{x}^{*} ; \mathrm{y}^{*} ; \mathrm{z}^{*}\right)=\mathrm{L}(\mathrm{x} ; \mathrm{y} ; \mathrm{z}) ; \mathrm{t}^{*}=\frac{\mathrm{L}^{2}}{\alpha} \mathrm{t} ; \overrightarrow{\mathrm{V}}^{*}=\frac{\alpha}{\mathrm{L}} \overrightarrow{\mathrm{V}} \\
& \mathrm{P}^{*}=\frac{\eta \alpha}{\mathrm{L}^{2}} \mathrm{P} ; \mathrm{T}^{*}-\mathrm{T}_{0}^{*}=\frac{\mu \alpha}{\rho_{0} \beta \mathrm{g}^{*} \mathrm{~L}^{3}} \mathrm{~T} ; \chi^{*}-\chi_{0}^{*}=\chi_{0}^{*} \chi
\end{aligned}
$$

Then, we can get from the equations (1)-(5) the following adimensional forms:

$$
\begin{aligned}
& \vec{\nabla} \cdot \vec{V}=0 \\
& \mathrm{P}_{\mathrm{r}}^{-1}\left[\frac{\partial \overrightarrow{\mathrm{V}}}{\partial \mathrm{t}}+(\overrightarrow{\mathrm{V}} \cdot \vec{\nabla}) \overrightarrow{\mathrm{V}}\right]=-\vec{\nabla}\left(\mathrm{P}+\mathrm{R}_{\mathrm{M}} \mathrm{z}\right)+\sqrt{\mathrm{T}_{\mathrm{A}}}\left(\overrightarrow{\mathrm{e}}_{\mathrm{x}}-\mathrm{u} \overrightarrow{\mathrm{e}}_{\mathrm{y}}\right) \\
& +\vec{\nabla}^{2} \vec{V}+\left[\left(1-\chi_{0}^{*}\right) T-R_{N} \chi-\chi_{0}^{*} \mathrm{~T} \chi\right] \overrightarrow{\mathrm{e}}_{\mathrm{z}} \\
& {\left[\frac{\partial \mathrm{T}}{\partial \mathrm{t}}+(\overrightarrow{\mathrm{V}} \cdot \vec{\nabla}) \mathrm{T}\right]=\vec{\nabla}^{2} \mathrm{~T}+\mathrm{R} \frac{\partial^{2} \mathrm{~T}}{\partial z^{2}}+\mathrm{R}_{\mathrm{a}}} \\
& +\mathrm{N}_{\mathrm{B}}\left[\mathrm{L}_{\mathrm{e}}^{-1} \vec{\nabla} \chi \cdot \vec{\nabla} \mathrm{T}+\mathrm{N}_{\mathrm{A}} \mathrm{L}_{\mathrm{e}}^{-1} \vec{\nabla} \mathrm{T} \cdot \vec{\nabla} \mathrm{T}\right] \\
& \frac{\partial \chi}{\partial \mathrm{t}}+(\overrightarrow{\mathrm{V}} \cdot \vec{\nabla}) \chi=\mathrm{L}_{\mathrm{e}}^{-1} \vec{\nabla}^{2} \chi+\mathrm{N}_{\mathrm{A}} \mathrm{L}_{\mathrm{e}}^{-1} \vec{\nabla}^{2} \mathrm{~T}
\end{aligned}
$$

Such that:

$$
\begin{gathered}
\mathrm{P}_{\mathrm{r}}=\frac{\mu}{\rho_{0} \alpha} ; \mathrm{R}_{\mathrm{M}}=\frac{\left[\rho_{0}\left(1-\chi_{0}^{*}\right)+\rho_{\mathrm{p}} \chi_{0}^{*}\right] \mathrm{g}^{*} \mathrm{~L}^{3}}{\mu \alpha} ; \mathrm{T}_{\mathrm{A}}=\left(\frac{2 \rho_{0} \Omega^{*} \mathrm{~L}^{2}}{\mu}\right)^{2} \\
\mathrm{R}_{\mathrm{N}}=\frac{\left(\rho_{\mathrm{p}}-\rho_{0}\right) \chi_{0}^{*} \mathrm{~g}^{*} \mathrm{~L}^{3}}{\mu \alpha} ; \alpha=\frac{\kappa}{(\rho c)} ; \mathrm{N}_{\mathrm{B}}=\frac{(\rho c)_{\mathrm{p}}}{(\rho c)} \chi_{0}^{*} ; \mathrm{L}_{\mathrm{e}}=\frac{\alpha}{\mathrm{D}_{\mathrm{B}}} \\
\mathrm{N}_{\mathrm{A}}=\frac{\mathrm{D}_{\mathrm{T}} \alpha \mu}{\mathrm{D}_{\mathrm{B}} \chi_{0}^{*} \mathrm{~T}_{0}^{*} \rho_{0} \beta \mathrm{g}^{*} \mathrm{~L}^{3}} ; \mathrm{R}=\frac{16 \sigma_{\mathrm{e}} \mathrm{T}_{0}^{* 3}}{3 \beta_{\mathrm{R}} \kappa} ; \mathrm{R}_{\mathrm{a}}=\frac{\rho_{0} \beta \mathrm{g}^{*} \mathrm{~L}^{5} \mathrm{Q}_{\mathrm{s}}^{*}}{\mu \alpha \kappa}
\end{gathered}
$$

\section{STABILITY ANALYSIS}

\subsection{The basic solutions}

The basic solution of our problem is a quiescent thermal equilibrium state, it's assumed to be independent of time where the equilibrium variables are varying only in the $\mathrm{z}$ direction, therefore:

$$
\begin{array}{cccc}
\vec{V}_{\mathrm{b}}=\overrightarrow{0} & & \\
\mathrm{~T}_{\mathrm{b}}=0 & ; \frac{\mathrm{d} \chi_{\mathrm{b}}}{\mathrm{dz}}+\mathrm{N}_{\mathrm{A}} \frac{\mathrm{dT}_{\mathrm{b}}}{\mathrm{dz}}=0 & \text { at } & \mathrm{z}=0 \\
\mathrm{~T}_{\mathrm{b}}=0 ; & ; \frac{\mathrm{d} \chi_{\mathrm{b}}}{\mathrm{dz}}+\mathrm{N}_{\mathrm{A}} \frac{\mathrm{dT}}{\mathrm{dz}}=0 & \text { at } & \mathrm{z}=1
\end{array}
$$

If we introduce the precedent results into equations (7)-(9), we obtain:

$$
\begin{gathered}
\vec{\nabla}\left[\mathrm{P}_{\mathrm{b}}+\mathrm{R}_{\mathrm{M}} \mathrm{z}\right]=\left[\left(1-\chi_{0}^{*}\right) \mathrm{T}_{\mathrm{b}}-\mathrm{R}_{\mathrm{N}} \chi_{\mathrm{b}}-\chi_{0}^{*} \mathrm{~T}_{\mathrm{b}} \chi_{\mathrm{b}}\right] \overrightarrow{\mathrm{e}}_{\mathrm{z}} \\
\frac{\mathrm{d}^{2} \mathrm{~T}_{\mathrm{b}}}{\mathrm{dz}^{2}}+\mathrm{R} \frac{\mathrm{d}^{2} \mathrm{~T}_{\mathrm{b}}}{\mathrm{dz}^{2}}+\mathrm{R}_{\mathrm{a}}+\mathrm{N}_{\mathrm{B}} \mathrm{L}_{\mathrm{e}}^{-1}\left(\frac{\mathrm{d} \chi_{\mathrm{b}}}{\mathrm{dz}} \frac{\mathrm{dT}_{\mathrm{b}}}{\mathrm{dz}}\right)+\mathrm{N}_{\mathrm{A}} \mathrm{N}_{\mathrm{B}} \mathrm{L}_{\mathrm{e}}^{-1}\left(\frac{\mathrm{dT}_{\mathrm{b}}}{\mathrm{dz}}\right)^{2}=0
\end{gathered}
$$

$$
\frac{\mathrm{d}^{2} \chi_{\mathrm{b}}}{\mathrm{dz^{2 }}}+\mathrm{N}_{\mathrm{A}} \frac{\mathrm{d}^{2} \mathrm{~T}_{\mathrm{b}}}{\mathrm{dz}^{2}}=0
$$

After using the boundary conditions (11) and (12), we can integrate the equation (15) between 0 and $z$ for obtaining:

$$
\chi_{\mathrm{b}}=-\mathrm{N}_{\mathrm{A}} \mathrm{T}_{\mathrm{b}}+\chi_{0}
$$

Where $\chi_{0}=\left(\chi^{*}(0)-\chi_{0}^{*}\right) / \chi_{0}^{*}$ is the relative nanoparticle volume fraction at $z=0$.

If we take into account the expression (16), we can get after simplification of the equation (14):

$$
\frac{\mathrm{d}^{2} \mathrm{~T}_{\mathrm{b}}}{\mathrm{dz}^{2}}=-\frac{\mathrm{R}_{\mathrm{a}}}{1+\mathrm{R}}
$$

Finally, we obtain after an integrating of the equation (17) between 0 and $z$ :

$$
\begin{gathered}
\mathrm{T}_{\mathrm{b}}=\frac{\mathrm{R}_{\mathrm{a}}}{2(1+\mathrm{R})} \mathrm{z}(1-\mathrm{z}) \\
\chi_{\mathrm{b}}=-\frac{\mathrm{N}_{\mathrm{A}} \mathrm{R}_{\mathrm{a}}}{2(1+\mathrm{R})} \mathrm{z}(1-\mathrm{z})+\chi_{0}
\end{gathered}
$$

\subsection{Perturbation of the basic state}

For analyzing the stability of the system, we superimpose infinitesimal perturbations on the basic solutions as follows:

$\mathrm{T}=\mathrm{T}_{\mathrm{b}}+\mathrm{T}^{\prime} ; \overrightarrow{\mathrm{V}}=\overrightarrow{\mathrm{V}}_{\mathrm{b}}+\overrightarrow{\mathrm{V}}^{\prime} ; \mathrm{P}=\mathrm{P}_{\mathrm{b}}+\mathrm{P}^{\prime} ; \chi=\chi_{\mathrm{b}}+\chi^{\prime}$

After introducing the expressions (20) into equations (6)-(9), we obtain the following linearized equations:

$$
\begin{gathered}
\vec{\nabla} \cdot \overrightarrow{\mathrm{V}^{\prime}}=0 \\
\mathrm{P}_{\mathrm{r}}^{-1} \frac{\partial \overrightarrow{\mathrm{V}}^{\prime}}{\partial \mathrm{t}}=-\vec{\nabla} \mathrm{P}^{\prime}+\sqrt{\mathrm{T}_{\mathrm{A}}}\left(\mathrm{v}^{\prime} \overrightarrow{\mathrm{e}}_{\mathrm{x}}-\mathrm{u}^{\prime} \overrightarrow{\mathrm{e}}_{\mathrm{y}}\right)+\vec{\nabla}^{2} \overrightarrow{\mathrm{V}}^{\prime} \\
+\left(\mathrm{f}_{1} \mathrm{~T}^{\prime}+\mathrm{f}_{2} \chi^{\prime}\right) \overrightarrow{\mathrm{e}}_{\mathrm{z}} \\
\frac{\partial \mathrm{T}^{\prime}}{\partial \mathrm{t}}+\mathrm{f}_{3} \mathrm{w}^{\prime}=\vec{\nabla}^{2} \mathrm{~T}^{\prime}+\mathrm{R} \frac{\partial^{2} \mathrm{~T}^{\prime}}{\partial \mathrm{z}^{2}}+\mathrm{f}_{4} \frac{\partial \mathrm{T}^{\prime}}{\partial \mathrm{z}}+\mathrm{f}_{5} \frac{\partial \chi^{\prime}}{\partial \mathrm{z}} \\
\frac{\partial \chi^{\prime}}{\partial \mathrm{t}}+\mathrm{f}_{6} \mathrm{w}^{\prime}=\mathrm{L}_{\mathrm{e}}^{-1} \vec{\nabla}^{2} \chi^{\prime}+\mathrm{N}_{\mathrm{A}} \mathrm{L}_{\mathrm{e}}^{-1} \vec{\nabla}^{2} \mathrm{~T}^{\prime}
\end{gathered}
$$

Such that:

$$
\begin{aligned}
& \mathrm{f}_{1}=1-\chi_{0}^{*}\left(1+\chi_{\mathrm{b}}\right) ; \mathrm{f}_{2}=-\left(\mathrm{R}_{\mathrm{N}}+\chi_{0}^{*} \mathrm{~T}_{\mathrm{b}}\right) ; \mathrm{f}_{3}=\mathrm{DT}_{\mathrm{b}} ; \mathrm{D}=\mathrm{d} / \mathrm{dz} \\
& \mathrm{f}_{4}=\mathrm{N}_{\mathrm{B}} \mathrm{L}_{\mathrm{e}}^{-1} \mathrm{D}\left(\chi_{\mathrm{b}}+2 \mathrm{~N}_{\mathrm{A}} \mathrm{T}_{\mathrm{b}}\right) ; \mathrm{f}_{5}=\mathrm{N}_{\mathrm{B}} \mathrm{L}_{\mathrm{e}}^{-1} \mathrm{DT}_{\mathrm{b}} ; \mathrm{f}_{6}=\mathrm{D} \chi_{\mathrm{b}} \\
& \text { After application of the curl operator twice to the equation }
\end{aligned}
$$
(22) and using the equation (21), we obtain the following equations:

$$
\begin{gathered}
\mathrm{P}_{\mathrm{r}}^{-1} \frac{\partial \mathrm{F}^{\prime}}{\partial \mathrm{t}}=\vec{\nabla}^{2} \mathrm{~F}^{\prime}+\sqrt{\mathrm{T}_{\mathrm{A}}} \frac{\partial \mathrm{w}^{\prime}}{\partial \mathrm{z}} \\
\mathrm{P}_{\mathrm{r}}^{-1} \frac{\partial}{\partial \mathrm{t}} \vec{\nabla}^{2} \mathrm{w}^{\prime}=\vec{\nabla}^{4} \mathrm{w}^{\prime}-\sqrt{\mathrm{T}_{\mathrm{A}}} \frac{\partial \mathrm{F}^{\prime}}{\partial \mathrm{z}}+\left(\mathrm{f}_{1} \vec{\nabla}_{2}^{2} \mathrm{~T}^{\prime}+\mathrm{f}_{2} \vec{\nabla}_{2}^{2} \chi^{\prime}\right)
\end{gathered}
$$

Where $\vec{\nabla}_{2}^{2}=\left(\frac{\partial^{2}}{\partial \mathrm{x}^{2}}\right)+\left(\frac{\partial^{2}}{\partial \mathrm{y}^{2}}\right)$ and $\mathrm{F}^{\prime}=\left(\frac{\partial \mathrm{v}^{\prime}}{\partial \mathrm{x}}-\frac{\partial \mathrm{u}^{\prime}}{\partial \mathrm{y}}\right)$.

Analyzing the disturbances into normal modes, we can simplify the equations (23)-(26) by assuming that the perturbation quantities are of the form:

$\left(\mathrm{w}^{\prime}, \mathrm{T}^{\prime}, \chi^{\prime}, \mathrm{F}^{\prime}\right)=(w(\mathrm{z}), \mathcal{T}(\mathrm{z}), \chi(\mathrm{z}), \mathcal{F}(\mathrm{z})) e^{\left[\mathrm{i}\left(\mathrm{a}_{\mathrm{x}} \mathrm{x}+\mathrm{a}_{\mathrm{y}} \mathrm{y}\right)+\mathrm{nt}\right]}$

After introducing the expressions (27) into equations (23)(26), we obtain:

$$
\begin{gathered}
\mathrm{P}_{\mathrm{r}}^{-1} \mathrm{n} \mathcal{F}=\left(\mathrm{D}^{2}-\mathrm{a}^{2}\right) \mathcal{F}+\sqrt{\mathrm{T}_{\mathrm{A}} \mathrm{D} w} \\
\mathrm{P}_{\mathrm{r}}^{-1} \mathrm{n}\left(\mathrm{D}^{2}-\mathrm{a}^{2}\right) w=\left(\mathrm{D}^{2}-\mathrm{a}^{2}\right)^{2} w-\sqrt{\mathrm{T}_{\mathrm{A}}} \mathrm{D} \mathcal{F} \\
-\mathrm{a}^{2}\left(\mathrm{f}_{1} \mathcal{T}+\mathrm{f}_{2} \mathcal{X}\right) \\
\mathrm{n} \mathcal{T}+\mathrm{f}_{3} w=\left(\mathrm{D}^{2}-\mathrm{a}^{2}\right) \mathcal{T}+\mathrm{RD}^{2} \mathcal{T}+\mathrm{f}_{4} \mathrm{D} \mathcal{T}+\mathrm{f}_{5} \mathrm{D} \mathcal{X} \\
\mathrm{n} \mathcal{X}+\mathrm{f}_{6} w=\mathrm{N}_{\mathrm{A}} \mathrm{L}_{\mathrm{e}}^{-1}\left(\mathrm{D}^{2}-\mathrm{a}^{2}\right) \mathcal{T}+\mathrm{L}_{\mathrm{e}}^{-1}\left(\mathrm{D}^{2}-\mathrm{a}^{2}\right) \mathcal{X}
\end{gathered}
$$

Where $a=\sqrt{a_{x}^{2}+a_{y}^{2}}$ is the dimensionless resultant wave number.

In the framework of the Oberbeck-Boussinesq approximations, we can neglect the terms coming from the product of the temperature and the volumetric fraction of 
nanoparticles in equation (7), if we suppose also that we are in the case of small temperature gradients in a dilute suspension of nanoparticles, we can consider that $f_{1} \approx 1$ and $f_{2} \approx-R_{N}$ in the equation (29).

The equations (28)-(31) will be solved subject to the following rigid-rigid boundary conditions:

$$
w=\mathrm{D} w=\mathcal{F}=\mathcal{T}=\mathrm{D}\left(\mathcal{X}+\mathrm{N}_{\mathrm{A}} \mathcal{T}\right)=0 \quad \text { at } \quad \mathrm{z}=0 ; 1
$$

\section{METHOD OF SOLUTION}

\subsection{The numerical method}

Very recently, Nield and Kuznetsov [21] and Agarwal [22] observed that the oscillatory convection is ruled out for the nanofluids with this new type of boundary conditions due to very large nanofluid Lewis number, so the stationary convection $(n=0)$ is the predominant mode. Hence, the equations (28)-(31) become:

$$
\begin{gathered}
\sqrt{\mathrm{T}_{\mathrm{A}}} \mathrm{D} w+\left(\mathrm{D}^{2}-\mathrm{a}^{2}\right) \mathcal{F}=0 \\
\left(\mathrm{D}^{2}-\mathrm{k}^{2}\right)^{2} w-\mathrm{a}^{2} \mathrm{f}_{1} \mathcal{T}-\mathrm{a}^{2} \mathrm{f}_{2} X-\sqrt{\mathrm{T}_{\mathrm{A}}} \mathrm{D} \mathcal{F}=0 \\
\mathrm{f}_{3} w-\left[(1+\mathrm{R}) \mathrm{D}^{2}+\mathrm{f}_{4} \mathrm{D}-\mathrm{a}^{2}\right] \mathcal{T}-\mathrm{f}_{5} \mathrm{D} \mathcal{X}=0 \\
\mathrm{f}_{6} w-\mathrm{N}_{\mathrm{A}} \mathrm{L}_{\mathrm{e}}^{-1}\left(\mathrm{D}^{2}-\mathrm{a}^{2}\right) \mathcal{T}-\mathrm{L}_{\mathrm{e}}^{-1}\left(\mathrm{D}^{2}-\mathrm{a}^{2}\right) \mathcal{X}=0
\end{gathered}
$$

We can solve the equations (33)-(36) which are subjected to the conditions (32), by making a suitable change of variables that makes the number of variables equal to the number of boundary conditions to obtain a set of ten first order ordinary differential equations which we can write it in the following form:

$$
\frac{d}{d z} u_{i}(z)=a_{i j} u_{j}(z) ; 1 \leq i, j \leq 10
$$

The solution of the system (37) in matrix notation can be written as follows:

$$
\mathrm{U}=\mathrm{BC}
$$

Where:

$$
\mathrm{B}=\left(\left(\mathrm{b}_{\mathrm{ij}}(\mathrm{z})\right)_{\substack{1 \leq \mathrm{i} i \mathrm{10} \\ 1 \leq \mathrm{j} \leq 10}}\right) ; \mathrm{U}=\left(\left(\mathrm{u}_{\mathrm{i}}(\mathrm{z})\right)_{1 \leq \mathrm{i} \leq 10}\right)^{\mathrm{T}} ; \quad \mathrm{C}=\left(\left(\mathrm{c}_{\mathrm{j}}\right)_{1 \leq \mathrm{j} \leq 10}\right)^{\mathrm{T}}
$$

If we assume that the matrix $B$ is written in the following form:

$$
\mathrm{B}=\left(\left(\mathrm{u}_{\mathrm{i}}^{\mathrm{j}}(\mathrm{z})\right)_{\substack{1 \leq \mathrm{i} \leq 10 \\ 1 \leq j \leq 10}}\right)
$$

Therefore, the use of five boundary conditions at $z=0$, allows us to write each variable $u_{i}(z)$ as a linear combination for five functions $u_{i}^{j}(z)$, such that:

$$
b_{i j}(0)=u_{i}^{j}(0)=\delta_{i j}
$$

Where $\delta_{i j}$ is the Kronecker delta symbol.

After introducing the new expressions of the variables $u_{i}(z)$ in the system (37), we will obtain the following equations:

$$
\frac{\mathrm{d}}{\mathrm{dz}} \mathrm{u}_{\mathrm{i}}^{\mathrm{j}}(\mathrm{z})=\mathrm{a}_{\mathrm{il}} \mathrm{u}_{\mathrm{l}}^{\mathrm{j}}(\mathrm{z}) ; 1 \leq \mathrm{i}, \mathrm{l}, \mathrm{j} \leq 10
$$

For each value of $j$, we must solve a set of ten first order ordinary differential equations which are subjected to the initial conditions (40), by approaching the variables $u_{i}^{j}(z)$ with power series defined in the interval $[0,1]$ and truncated at the order $N$, such that:

$$
u_{i}^{j}(z)=\sum_{p=0}^{p=N} d_{p}^{i, j} z^{p}
$$

A linear combination of the solutions $u_{i}^{j}(z)$ satisfying the boundary conditions (32) at $z=1$ leads to a homogeneous algebraic system for the coefficients of the combination. A necessary condition for the existence of nontrivial solution is the vanishing of the determinant which can be formally written as:

$$
f\left(R_{a}, a, T_{A}, R, N_{B}, L_{e}, R_{N}, N_{A}\right)=0
$$

If we give to each control parameter $\left(T_{A}, R, N_{B}, L_{e}, R_{N}, N_{A}\right)$ its value, we can plot the neutral curve of the stationary convection by the numerical research of the smallest real positive value of the thermal Rayleigh number $R_{a}$ which corresponds to a fixed wave number $a$ and verifies the dispersion relation (43). After that, we will find a set of points $\left(a, R_{a}\right)$ which help us to plot our curve and find the critical value $\left(a_{c}, R_{a c}\right)$ which characterizes the onset of the convective stationary instability, this critical value represents the minimum value of the obtained curve.

\subsection{Validation of the method}

The main aim of our study consists to study the influence of a uniform rotation on the convective instability which is induced by a purely internal heating in a confined medium filled of a Newtonian nanofluid layer (water + alumina) for the rigid-rigid boundaries in the case where the radiation heat transfer mode is taken into consideration . Our study shows that the thermal stability of Newtonian nanofluids depends on six parameters: $T_{A}, R, N_{B}, L_{e}, R_{N}$ and $N_{A}$. To validate our

\begin{tabular}{|c|c|c|c|c|}
\hline \multicolumn{3}{|c|}{ Present study } & \multicolumn{2}{|c|}{ Yu and Shih } \\
\hline $\mathrm{N}$ & $a_{c}$ & $\mathrm{R}_{\mathrm{ac}}$ & $a_{c}$ & $\mathrm{R}_{\mathrm{ac}}$ \\
\hline 35 & 3.99 & 37325.80 & & \\
\hline 36 & 3.99 & 37324.96 & & \\
\hline 37 & 3.99 & 37325.24 & & \\
\hline 38 & 3.99 & 37325.15 & & \\
\hline 39 & 3.99 & 37325.18 & & \\
\hline 40 & 3.99 & 37325.17 & 4 & 37325 \\
\hline 41 & 3.99 & 37325.17 & & \\
\hline 42 & 3.99 & 37325.17 & & \\
\hline 43 & 3.99 & 37325.17 & & \\
\hline 44 & 3.99 & 37325.17 & & \\
\hline 45 & 3.99 & 37325.17 & & \\
\hline
\end{tabular}
method, we compared our results with those obtained by $\mathrm{Yu}$ and Shih [30] concerning the onset of convective instability which is induced by a purely internal heating in a non-rotating layer of a regular fluid confined between two isothermal rigid boundaries without taking into account the radiation heat transfer mode (Table 1) .To make this careful comparison , we must take into consideration the following restrictions :

$$
\mathrm{T}_{\mathrm{A}}=\mathrm{R}=\mathrm{N}_{\mathrm{B}}=\mathrm{L}_{\mathrm{e}}^{-1}=\mathrm{R}_{\mathrm{N}}=\mathrm{N}_{\mathrm{A}}=0
$$

Table 1: The comparison of critical values of Rayleigh number and the corresponding wave number with $\mathrm{Yu}$ and Shih [30] for the regular fluids in the case where $T_{A}=R=0$ for the rigid-rigid boundaries.

\subsection{Convergence of the numerical method}

The convergence of our method is tested for the regular fluids and also for a reference nanofluid $\left(\mathrm{H}_{2} \mathrm{O}+\mathrm{Al}_{2} \mathrm{O}_{3}\right)$ in different cases of the Taylor number $\left(T_{A}=200 ; 600 ; 1000\right)$ and for a fixed value of the radiation parameter: $R=0.6$ (Fig 2).

According to the thermo-physical properties of the reference nanofluid which are given by Buongiorno [1] in the Table 2, we find for the considered nanofluid:

$\mathrm{N}_{\mathrm{B}}=7.75 \times 10^{-3} ;$ Le $=5000 ; \mathrm{R}_{\mathrm{N}}=1.03 \times 10^{-1} ; \mathrm{N}_{\mathrm{A}}=24.18$ 
Table 2: Some properties of $\left(\mathrm{H}_{2} \mathrm{O}+\mathrm{Al}_{2} \mathrm{O}_{3}\right)$ in the case where $\chi_{0}^{*}=0.01, \mathrm{~T}_{0}^{*}=300 \mathrm{~K}$ such that $\mathrm{d}_{\mathrm{p}}=10 \mathrm{~nm}, \mathrm{~L}=4.1 \times 10^{-5} \mathrm{~m}$ and $\rho_{0}=10^{3} \mathrm{~kg} / \mathrm{m}^{3}$.

\begin{tabular}{|c|c|c|c|c|c|c|c|c|}
\hline$\mu($ Pa.s $)$ & $\kappa(\mathrm{W} / \mathrm{m} . \mathrm{K})$ & $\alpha\left(\mathrm{m}^{2} / \mathrm{s}\right)$ & $\mathrm{D}_{\mathrm{B}}\left(\mathrm{m}^{2} / \mathrm{s}\right)$ & $\mathrm{D}_{\mathrm{T}}\left(\mathrm{m}^{2} / \mathrm{s}\right)$ & $\beta\left(\mathrm{K}^{-1}\right)$ & $\rho_{\mathrm{p}}\left(\mathrm{kg} / \mathrm{m}^{3}\right)$ & $(\rho \mathrm{c})\left(\mathrm{J} / \mathrm{m}^{3}\right)$ & $(\rho \mathrm{c})_{\mathrm{p}}\left(\mathrm{J} / \mathrm{m}^{3}\right)$ \\
\hline $10^{-3}$ & 1 & $2 \times 10^{-7}$ & $4 \times 10^{-11}$ & $6 \times 10^{-11}$ & $6 \times 10^{-3}$ & $4 \times 10^{3}$ & $4 \times 10^{6}$ & $3.1 \times 10^{6}$ \\
\hline
\end{tabular}
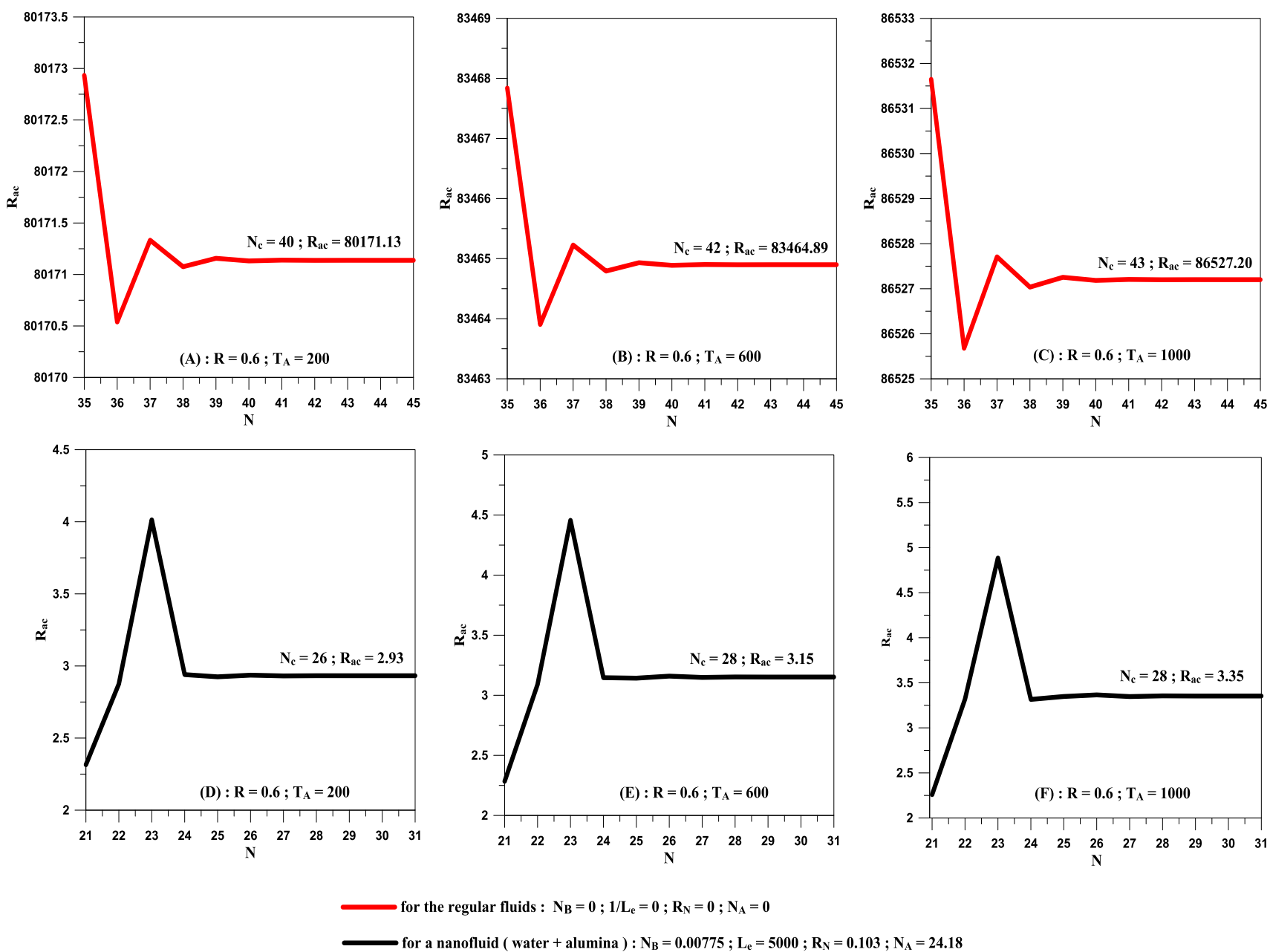

Fig 2: Plot of $R_{a c}$ as a function of $N$ for different values of $T_{A}$ for the regular fluids and the reference nanofluid.

The above figure (Fig 2) shows that the stationary instability threshold of the regular fluids or of the reference nanofluid depends implicitly of the truncation order $N$ and of the Taylor number $T_{A}$ such that the truncation order $N_{C}$ which corresponds at the convergence of our method is determined, when the absolute value of the difference between the critical thermal Rayleigh numbers $R_{a c}\left(N_{c}+1\right)$ and $R_{a c}\left(N_{c}\right)$ is of the order of $10^{-3}$, where $R_{a c}\left(N_{c}\right)$ and $R_{a c}\left(N_{c}+1\right)$ are the critical thermal Rayleigh numbers which correspond to the truncation orders $N_{c}$ and $N_{c}+1$ respectively, such that $R_{a c}=R_{a c}\left(N_{c}\right)$.

The numerical study of the convergence of the used method shows that to ensure the accuracy of our obtained critical values, we must take as truncation order $N_{c}=43$ for the regular fluids and $N_{c}=28$ for the reference nanofluid, when we want to vary the values of the Taylor number $T_{A}$ from 0 to 1000 .

\section{RESULTS AND DISCUSSION}

To study the effect of a parameter $\left(R, N_{B}, L_{e}, R_{N}, N_{A}\right)$ on the onset of the convective instability in a rotating medium filled of a Newtonian nanofluid layer $\left(\mathrm{H}_{2} \mathrm{O}+\mathrm{Al}_{2} \mathrm{O}_{3}\right)$ for the rigid-rigid boundaries in the case where the thermal radiation is taken into consideration and the convective instability is induced by a purely internal heating, we must fix the others and determine the variation of the critical Rayleigh number $R_{a c}$ and the critical wave number $a_{c}$ as a function of the Taylor number $T_{A}$ in the interval $[0,1000]$ for different values of this parameter and then compare the obtained results with those of the regular fluids (Figs 3-8). To ensure the accuracy in this study, we will take as truncation order : $N_{c}=28$ for the studied nanofluids and $N_{c}=43$ for the regular fluids. 

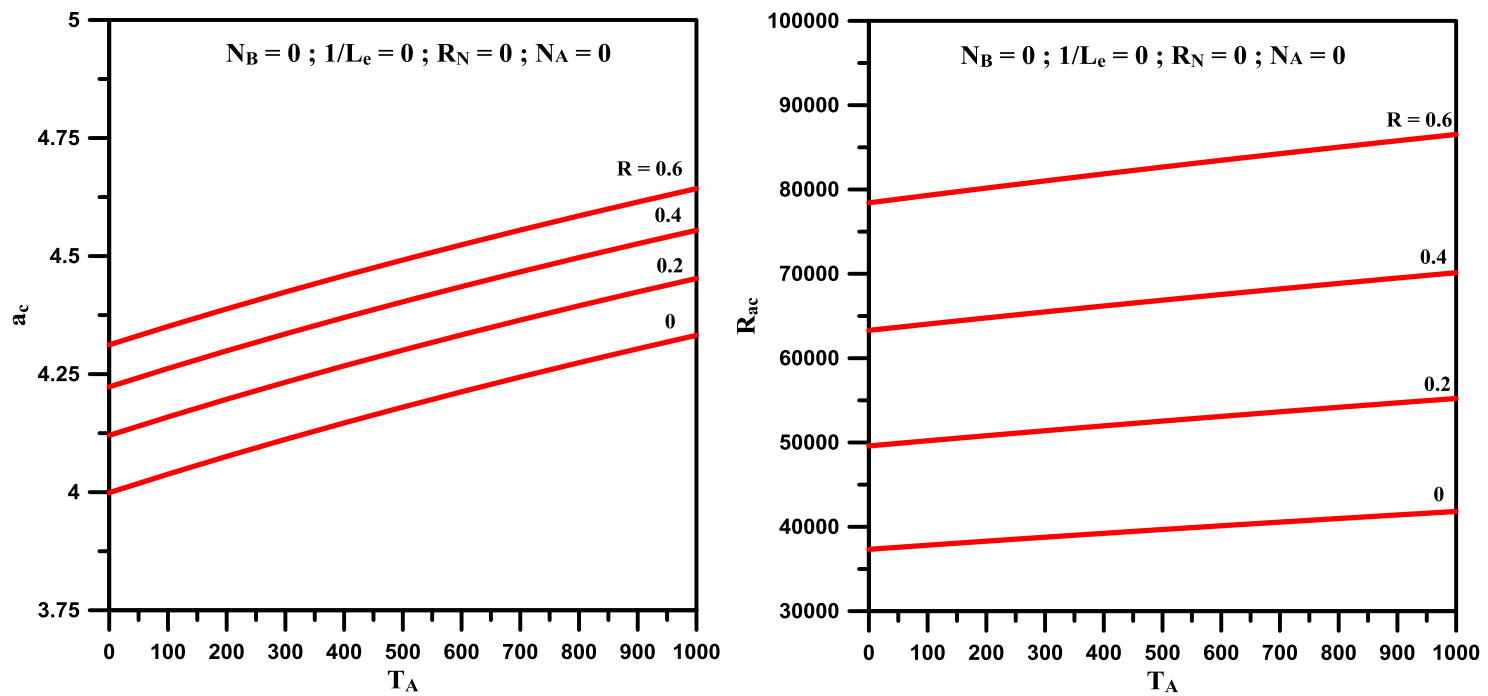

Fig 3: Plot of $a_{c}$ and $R_{a c}$ as a function of $T_{A}$ for different values of $R$ for the regular fluids.
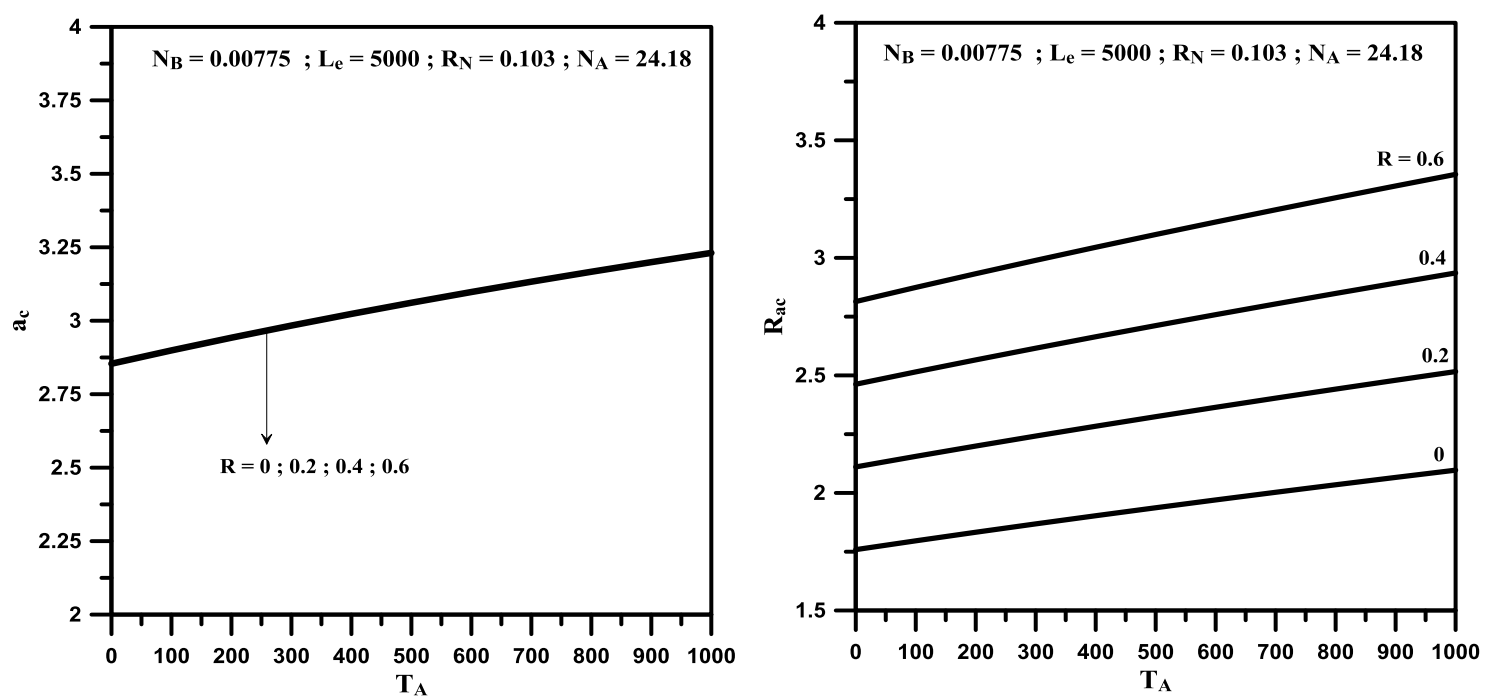

Fig 4: Plot of $a_{c}$ and $R_{a c}$ as a function of $T_{A}$ for different values of $R$ for a reference nanofluid.
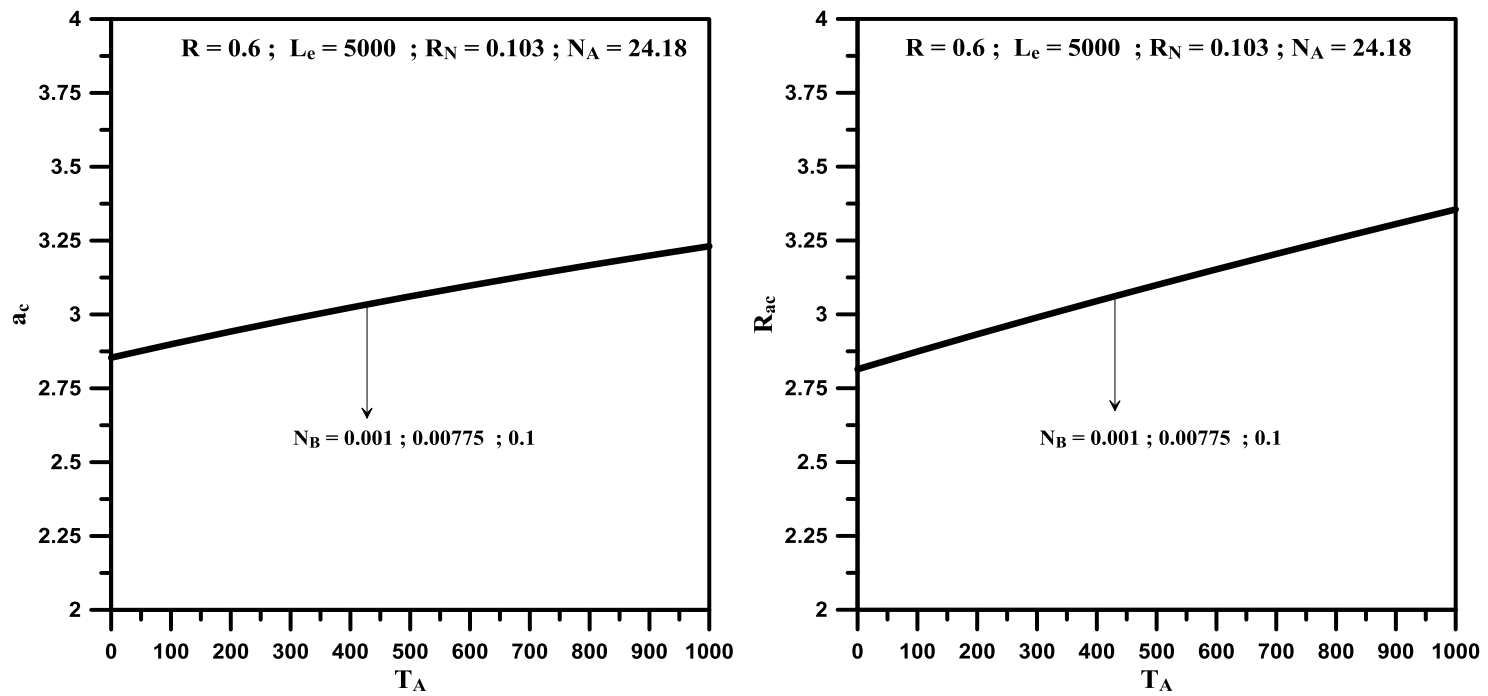

Fig 5 : Plot of $a_{c}$ and $R_{a c}$ as a function of $T_{A}$ for different values of $N_{B}$ for the nanofluids. 

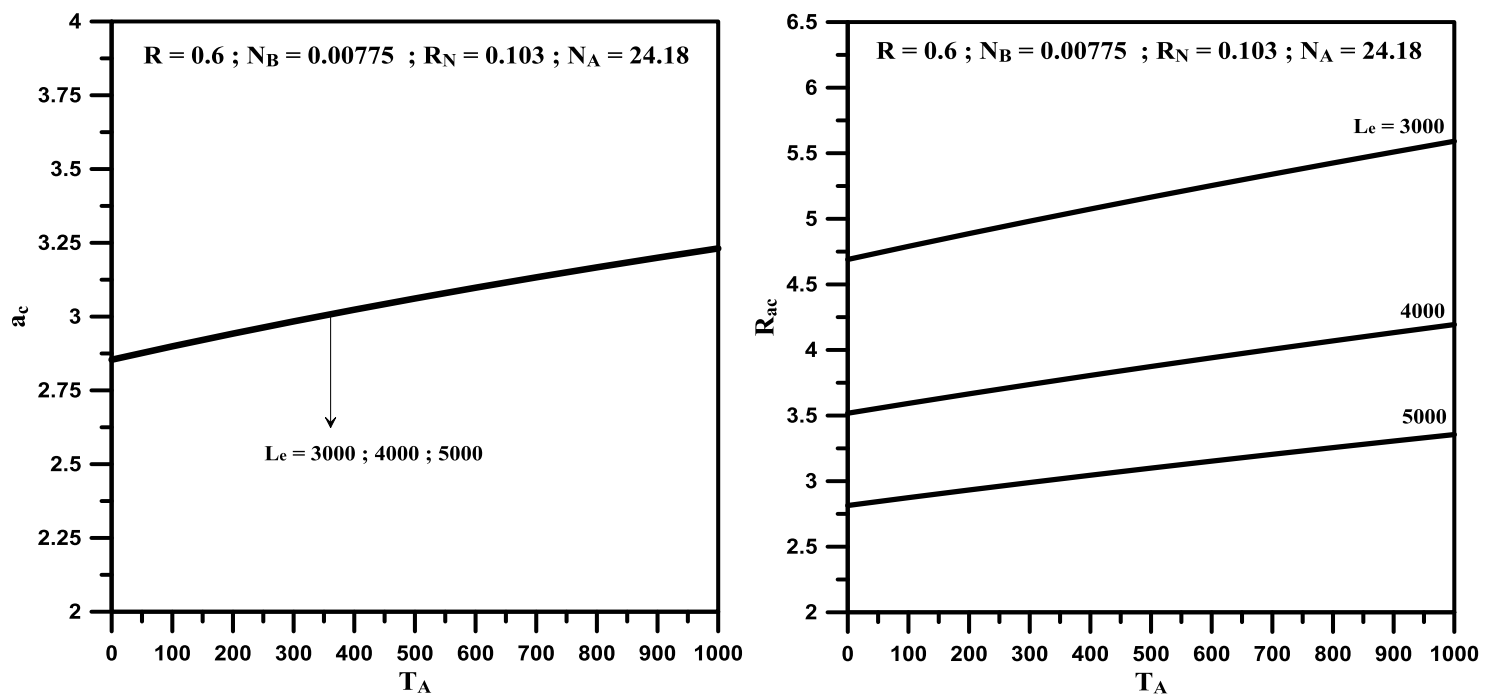

Fig 6 : Plot of ac and $R_{a c}$ as a function of $T_{A}$ for different values of $L_{e}$ for the nanofluids.
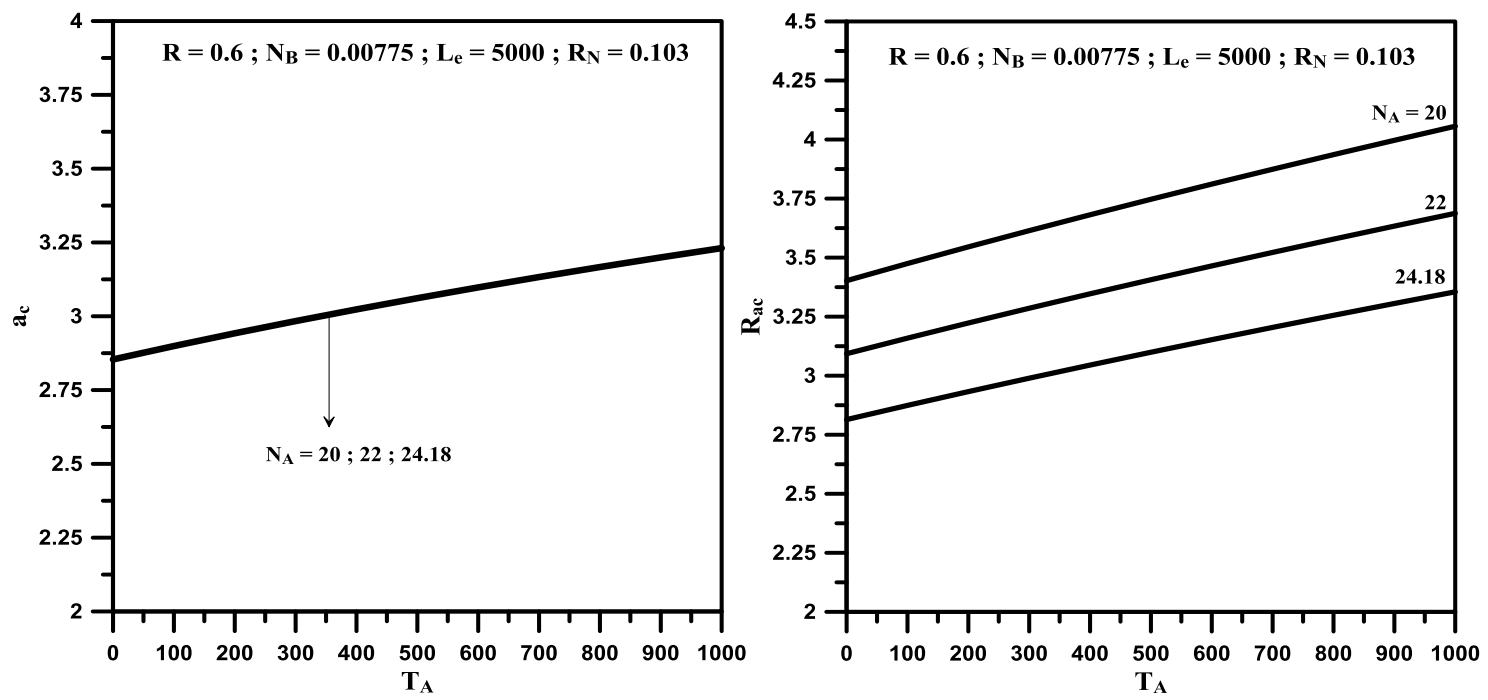

Fig 7: Plot of $a_{c}$ and $R_{a c}$ as a function of $T_{A}$ for different values of $N_{A}$ for the nanofluids.
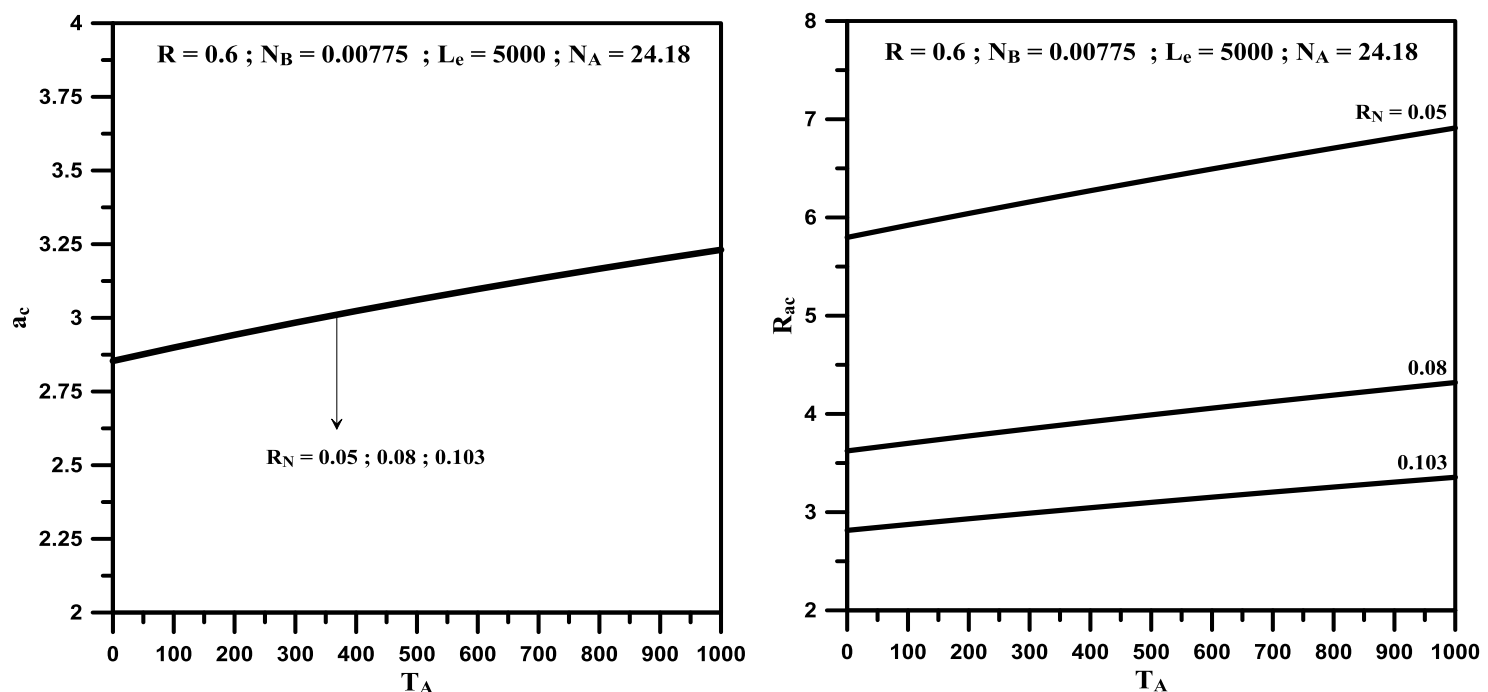

Fig 8 : Plot of $a_{c}$ and $R_{a c}$ as a function of $T_{A}$ for different values of $R_{N}$ for the nanofluids. 
In the present work concerning the study of the influence of a uniform rotation on the convective instability induced by a purely internal heating in the case where the thermal radiation is taken into consideration, we conclude generally that the presence of the nanoparticles in a base fluid can return the heat source strength $Q_{s}^{*}$ as a driving force which produces the onset of the convection in the gravity field for a configuration where the temperature on the top rigid boundary is equal that of the bottom. The influence of the thermal radiation parameter $R$ on the convective instability for the regular fluids and the nanofluids is shown on Figs $\mathbf{3}$ and $\mathbf{4}$, such that an increase in the thermal radiation parameter $R$ will produce also an increase in both critical values $R_{a c}$ and $a_{c}$ for the regular fluids (Fig 3), and an increase in thermal Rayleigh number $R_{a c}$ without any change in the critical wave number $a_{c}$ for the nanofluids (Fig 4). The precedents results may be explained by the dominance of the thermal conduction over the radiation heat transfer in the case where the thermal radiation parameter $R$ is increased, such that in this case the medium releases a small amount of thermal energy for the buoyancy forces (driven forces promoting the onset of convection) compared with the case where the thermal radiation parameter $R$ is zero ,for this reason the size of convection cells remains unaffected by the thermal radiations in the case where a base fluid contains a volume fraction of nanoparticles .

The Figs 3 and $\mathbf{4}$ indicate also that the variation either in the critical thermal Rayleigh number $R_{a c}$ or in the critical wave number $a_{c}$ with the Taylor number $T_{A}$ is an increasing function for both studied cases (the regular fluids and the nanofluids), these results show that the presence of the Coriolis forces in the medium has a double effect, on the one hand it minimizes the effect of the buoyancy forces, on the other hand, it reduces the size of convection cells. Hence, the Taylor number $T_{A}$ has a stabilizing effect. The precedents figures and tables show also that the presence of the nanoparticles $\mathrm{Al}_{2} \mathrm{O}_{3}$ in a base fluid like water allows us to reduce tremendously the critical thermal Rayleigh number $R_{a c}$ , this result confirm that the regular fluids are more stable than the nanofluids.

The Fig 5 shows the effect of the modified particle-density increment $N_{B}$ on the onset of convection. From the precedent figure, we observe that the modified particle-density increment $N_{B}$ has no significant effect on the stability of nanofluids convection. This is happened due to the low value of the term $N_{B}$ which appears only in the perturbed energy equation (23) as a product with the inverse of the Lewis number $L_{e}$ near the temperature gradient and the volume fraction gradient of nanoparticles, so the effect of this parameter on the onset of convection in nanofluids will be very small which we can neglect it, such that for the majority of the nanofluids, we have : $N_{B} \sim 10^{-3}-10^{-1}, L_{e} \sim 10^{2}-10^{3}$ (Buongiorno [1], Nield and Kuznetsov [4] and I.S. Shivakumara et al. [24]).

The Figs 6 and 7 exhibit the effects of the Lewis number $L_{e}$ and the modified diffusivity ratio $N_{A}$ on the stability of the system. From the precedent figures, we find that both the Lewis number $L_{e}$ and the modified diffusivity ratio $N_{A}$ accelerate the onset of convection in a nanofluid layer. The modified diffusivity ratio $N_{A}$ appears in the equations (8) and (9) as a product with the inverse of the Lewis number $L_{e}$ where $N_{A} L_{e}^{-1}=D_{T} \mu / \chi_{0}^{*} T_{0}^{*} \rho_{0} \beta g^{*} L^{3}$ and $L_{e}=\alpha / D_{B}$, this important remark confirms that the thermophoresis of nanoparticles is a factor producing the thermal instability in nanofluids, such that an increase in the thermophoresis, increases also the Brownian motion of nanoparticles. The effect of the Lewis number $L_{e}$ on the onset of convection shows that to ensure the stability of the nanofluids we can use a nanofluid which is having a less thermal diffusivity. In this investigation we find also that the Lewis number $L_{e}$ and the modified diffusivity ratio $N_{A}$ have no significant effect on the critical wave number $a_{c}$.

The Fig 8 shows the effect of the concentration Rayleigh number $R_{N}$ on the stability curves as a function of the Taylor number $T_{A}$ for the fixed values of other governing parameters. It is observed that the critical thermal Rayleigh number $R_{a c}$ decreases with the concentration Rayleigh number $R_{N}$, indicating that the effect of the concentration Rayleigh number $R_{N}$ is to destabilize the system . The precedent result may be explained as an increase in the value of the concentration Rayleigh number $R_{N}$ increases also the volumetric fraction of nanoparticles $\chi_{0}^{*}$ and hence both the Brownian motion and the thermophoretic diffusion of nanoparticles increase. In this investigation, we find that the concentration Rayleigh number $R_{N}$ has no significant effect on the critical wave number $a_{c}$ and the regular fluids are more stable than the nanofluids, such that to ensure the stability of the nanofluids, we can use the less dense nanoparticles.

\section{CONCLUSIONS}

In this paper, we have examined the convective instability induced by a purely internal heating for a rotating nanofluid in the case where the thermal radiation is taken into consideration. The analysis has been performed for zero flux nanoparticles condition at rigid boundaries such that these latter are taken as isothermal. The resulting eigenvalue problem is solved analytically and numerically using the power series method (A.Wakif et al. [23,25,26,31]). The behavior of various parameters like the Taylor number $T_{A}$, the thermal radiation parameter $R$, the modified particledensity increment $N_{B}$, the Lewis number $L_{e}$, the modified diffusivity ratio $N_{A}$ and the concentration Rayleigh number $R_{N}$ on the onset of convection has been analysed and presented both numerically and graphically.

The principal results derived from the present analysis can be summarized as follows:

i. We can generate the convective instability thanks to an internal heat source which produces a constant volumetric heat with isothermal boundaries.

ii. The presence of the radiation heat transfer and the Coriolis forces allow us to stabilize the nanofluids, such that an increase either in the thermal radiation parameter $\mathrm{R}$ or in the Taylor number $T_{A}$ induces also an increase in the critical thermal Rayleigh number $R_{a c}$.

iii. To ensure the stability of the nanofluids, we can use a nanofluid which are having a less thermal diffusivity, a low concentration of nanoparticles or consisting of less dense nanoparticles.

iv. An increase in the volume fraction of nanoparticles increases also both the Brownian motion and the thermophoresis of nanoparticles which cause a destabilizing effect for the nanofluids.

v. The thermal radiation parameter $R$, the modified particle-density increment $N_{B}$, the Lewis number $L_{e}$, the modified diffusivity ratio $N_{A}$ and the concentration Rayleigh number $R_{N}$ have no effect on the critical wave number $a_{c}$. On the contrary, the 
presence of the rotation allows us to reduce the size of convection cells for both the nanofluids and the regular fluids.

vi. The modified particle-density increment $N_{B}$ has no significant effect on the convective instability for the nanofluids. Hence, we can neglect the contribution of this parameter in the energy equation.

vii. The regular fluids are more stable than the nanofluids.

viii. The presence of the radiation heat transfer allows us to reduce the size of convection cells for the regular fluids.

\section{ACKNOWLEDGMENTS}

The authors wish to express their very sincerely thanks to the reviewers for their valuable and lucid comments which have improved the paper appreciably.

\section{REFERENCES}

[1] J. Buongiorno, "Convective transport in nanofluids," Journal of Heat Transfer, vol.128, pp.240-250, 2006.

[2] D.Y. Tzou, "Thermal instability of nanofluids in natural convection," International Journal of Heat and Mass Transfer, vol.51, pp.2967-2979, 2008.

[3] D.Y. Tzou, "Instability of nanofluids in natural convection", ASME Journal of Heat Transfer, 130, 2008, 372-401.

[4] D.A. Nield and A.V. Kuznetsov, "The onset of convection in a horizontal nanofluid layer of finite depth," European Journal of Mechanics B/Fluids, vol.29, pp.217-233, 2010.

[5] Dhananjay Yadav, R. Bhargava and G.S. Agrawal, "Numerical solution of a thermal instability problem in a rotating nanofluid layer," International Journal of Heat and Mass Transfer, vol.63, pp.313-322, 2013.

[6] Dhananjay Yadav, R. Bhargava and G.S. Agrawal, "Thermal instability in a nanofluid layer with a vertical magnetic field," J .Eng. Math, vol.80, pp.147-164, 2013.

[7] Dhananjay Yadav, R. Bhargava, G. S. Agrawal, Gyeong S. Hwang, Jinho Lee and M. C. Kim, "Magnetoconvection in a rotating layer of nanofluid," Asia-Pac. J. Chem. Eng., DOI: 10.1002/apj.1796.

[8] R.M. Goody, "The influence of radiative transfer on cellular convection," J. of Fluid Mech., vol.1, pp.424$435,1956$.

[9] Vedat S. Arpaci, "Effect of thermal radiation on the laminar free convection from a heated vertical plate," International Journal of Heat and Mass Transfer, vol.11, pp.871-881, 1968.

[10] C. Christophorides and Stephen H. Davis, "Thermal instability with radiative transfer," Physics of Fluids, vol.13, pp.222-226, 1970.

[11] J. C. Bratis and J.R. Novotny, "Radiation-convection interaction in the boundary layer-regime of an enclosure," Int. J. Heat Mass Transfer, vol.17, pp. 365379, 1974.

[12] C. Chang, K. T. Kang and J.R. Lloyd, "Radiation-natural convection interaction in two-dimensional complex enclosure,” ASME J. Heat Transfer, vol.105, pp. 89-95, 1983.

[13] Wen-Mei Yang, "Thermal instability of a fluid layer induced by radiation," Numerical Heat Transfer, Part A, vol.17, pp. 365-376, 1990.

[14] F. Bdéoui and A. Soufiani, "The onset of RayleighBénard instability in molecular radiating gases," Physics of Fluids, vol.9, pp.3858- 3872, 1997.

[15] A. Raptis, "Radiation and free convection flow through a porous medium," Int. Comm. Heat Mass Transfer, vol.25, pp.289-295, 1998.

[16] M.A. Hossain, M. Kutibuddin and H. S. Takhar, "Radiation interaction on combined forced and free convection across a horizontal cylinder," Int. J. Applied Mechanics and Engineering, vol.4, pp.219-235, 1999.

[17] A. J. Chamkha, "Effects of heat absorption and thermal radiation on heat transfer in a fluid - particle flow past a surface in the presence of a gravity field," Int. J. Thermal Sciences,vol. 39,pp. 605-615, 2000.

[18] M. Anwar Hossain, Khalil Khanafer and Kambiz Vafai, "The effect of radiation on free convection flow of fluid with variable viscosity from a porous vertical plate," Int. J. Thermal Sciences, vol.40, pp. 115-124, 2001.

[19] Vincent E. Larson, "The effects of thermal radiation on dry convective instability," Dynamics of Atmospheres and Oceans, vol.34, pp. 45-71, 2001.

[20] P. Ganesan and P. Loganathan, "Radiation and mass transfer effects on flow of an incompressible viscous fluid past a moving vertical cylinder," International Journal of Heat and Mass Transfer, vol.45, pp.42814288,2002

[21] D.A. Nield and A.V. Kuznetsov, "Thermal instability in a porous medium layer saturated by a nanofluid: a revised model," International Journal of Heat and Mass Transfer, vol.68, pp.211-214, 2014

[22] Shilpi Agarwal, "Natural Convection in a Nanofluidsaturated rotating porous layer: a more realistic approach," Transp. Porous Med., vol.104, pp.581-592, 2014.

[23] A.Wakif , Z.Boulahia and R.Sehaqui, "A Realistic Approach for Studying the Effect of an Internal Heat Source on the Onset of Convection in a Newtonian Nanofluid Layer: rigid-rigid case," IOSR-Journal of Mathematics,vol.11,issue 5, Ver. IV, pp.21-30, 2015.

[24] I.S. Shivakumara, M. Dhananjaya and Chiu-On Ng, "Thermal convective instability in an Oldroyd-B nanofluid saturated porous layer," International Journal of Heat and Mass Transfer, vol.84, pp.167-177, 2015.

[25] A.Wakif and R.Sehaqui, "A Realistic Study of the Rayleigh-Bénard Problem in the Newtonian Nanofluids with a Uniform Heat Source: Free-Free Case," International journal of science and research (IJSR), vol.4, issue 10, p. 611-615, 2015.

[26] A.Wakif , Z.Boulahia and R.Sehaqui, "An accurate method to Study the Rayleigh-Bénard Problem in a Rotating Layer Saturated by a Newtonian Nanofluid," International Journal of Innovation and Scientific Research,vol.20, issue 1 ,no.1, pp.25-37, 2016. 
[27] Dhananjay Yadav, Jinho Lee and Hyung Hee Cho, "Brinkman convection induced by purely internal heating in a rotating porous medium layer saturated by a nanofluid," Powder Technology, vol.286, pp.592-601, 2015.

[28] M.A. Sheremet, I. Pop and M.M. Rahman, "Threedimensional natural convection in a porous enclosure filled with a nanofluid using Buongiorno's mathematical model," International Journal of Heat and Mass Transfer, vol.82, pp.396-405, 2015.

[29] R. Viskanta and R. J. Grosh, "Boundary layer in thermal radiation absorbing and emitting media," International
Journal of Heat and Mass Transfer, vol.5, pp.795-806, 1962.

[30] C. P. Yu and Y. D. Shih, "Thermal instability of an internally heated fluid layer in a magnetic field," Physics of Fluids, vol.23, pp.411-412, 1980.

[31] A. Wakif, Z. Boulahia, M. Zaydan, N.Yadil, and R.Sehaqui, "The Power Series Method to Solve a Magneto-Convection Problem in a Darcy-Brinkman Porous Medium Saturated by an Electrically Conducting Nanofluid Layer," International Journal of Innovation and Applied Studies,vol.14, issue 4,no.4, pp.1048-1065, 2016. 